\title{
REMARKS ON "WEAK LIMITS OF ALMOST INVARIANT PROJECTIONS" BY FOIAS, PASNICU AND VOICULESCU
}

\author{
MARCH T. BOEDIHARDJO
}

Abstract. Ultraproducts of operators are used to give simpler proofs of certain results in the paper "Weak limits of almost invariant projections" by Foias, Pasnicu and Voiculescu.

Mathematics subject classification (2010): 47A15, 47A58.

Keywords and phrases: Strong reductivity, ultraproducts of operators, Calkin representation, noncommutative Weyl-von Neumann Theorem, almost invariant projections.

\section{REFERENCES}

[1] C. Apostol, C. FoiAş ANd D. Voiculescu, On strongly reductive algebras, Rev. Roumaine Math. Pures Appl. 21 (1976), no. 6, 663-641.

[2] J. W. CALKIN, Two-sided ideals and congruences in the ring of bounded operators in Hilbert space, Ann. of Math. 42 (1941), 839-873.

[3] C. FoiAş, C. PASNicu AND D. VoiculesCU, Weak limits of almost invariant projections, J. Operator Theory 2 (1979) 79-93.

[4] K. J. HARRISON, Strongly reductive operators, Acta Sci. Math. 27 (1975), no. 3-4, 205-212.

[5] B. PRUNARU, Strongly reductive algebras are self-adjoint, J. Operator Theory 48 (2002) 615-619.

[6] D. Voiculescu, A non-commutative Weyl-von Neumann theorem, Rev. Roumaine Math. Pures Appl. 21 (1976), no. 1, 97-113. 